raises questions about protection from severe disease once neutralizing antibodies have waned to levels below those needed to block infection. In fact, studies have demonstrated that in the context of lower levels of neutralizing antibody responses, $\mathrm{CD} 8^{+}$ $T$ cells are critical for more rapid control of SARS-CoV-2 in animal models ${ }^{10}$. Although delaying the second dose results in clearly higher levels of neutralizing antibodies, the possibility of lowered $\mathrm{T}$ cell responses in this regimen should also be considered. Other key questions remain. What is the durability of these higher neutralizing antibody responses after the delayed dose? Does delaying the second dose also lead to a slower decrease in the levels of neutralizing antibody responses? Will these higher levels of neutralizing antibodies more rapidly curb the upper-respiratory airway transmission of divergent variants such as Omicron? It will also be important to evaluate the immunological basis for the improved humoral responses with delayed second dose and whether this effect would also apply to other mRNA vaccine strategies ${ }^{11-13}$ and protein-based constructs ${ }^{14}$. As previous studies have determined that germinal-center responses persist for several (7-15) weeks after vaccination in humans ${ }^{15}$, it will also be important to determine if delaying vaccine doses potentially leads to longer-lasting germinal-center responses and, as a result, more-mature and more-potent neutralizing antibodies at the monoclonal antibody level. This knowledge could have important implications for future pandemic vaccines. Ultimately, the fine tuning of vaccines for optimal immunity against SARS-CoV-2 and its variants will probably form part of the solution to one day end the COVID-19 pandemic.

\section{David R. Martinez $\mathbb{D}^{1 凶}$ and Eng Eong Ooi (iD) $2 \bowtie$ \\ ${ }^{1}$ Department of Epidemiology, The University of North Carolina at Chapel Hill, Chapel Hill, NC,}

USA. ${ }^{2}$ Programme in Emerging Infectious Diseases,
Duke-NUS Medical School, Singapore, Singapore.

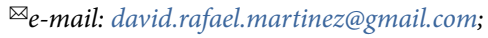

engeong.ooi@duke-nus.edu.sg

Published online: 21 February 2022

https://doi.org/10.1038/s41590-022-01143-Z

References

1. Payne, R. P. et al. Cell 184, 5699-5714.e5611 (2021).

2. Hall, V. G. et al. Nat. Immunol. https://doi.org/10.1038/s41590021-01126-6 (2022).

3. Guerrera, G. et al. Sci. Immunol. 6, eabl5344 (2021).

4. Baden, L. R. et al. N. Engl. J. Med. 384, 403-416 (2020).

5. Polack, F. P. et al. N. Engl. J. Med. 383, 2603-2615 (2020).

6. Lopez Bernal, J. et al. N. Engl. J. Med. 385, 585-594 (2021).

7. Kadire, S. R., Wachter, R. M. \& Lurie, N. N. Engl. J. Med. 384, e28 (2021).

8. Corbett, K. S. et al. Science 373, eabj0299 (2021).

9. Gilbert, P. B. et al. Science 375, 43-50 (2021).

10. McMahan, K. et al. Nature 590, 630-634 (2021).

11. de Alwis, R. et al. Mol. Ther. 29, 1970-1983 (2021).

12. Gebre, M. S. et al. Nature 601, 410-414 (2022).

13. Martinez, D. R. et al. Science 373, 991-998 (2021)

14. Heath, P. T. et al. N. Engl. J. Med. 385, 1172-1183 (2021).

15. Turner, J. S. et al. Nature 596, 109-113 (2021).

\title{
SARS-CoV-2 learned the 'Alpha'bet of immune evasion
}

\author{
Comparative analysis of SARS-CoV-2 isolates uncovers important mutations outside the spike gene that help the \\ Alpha variant to operate under the radar of innate immune surveillance.
}

\section{GuanQun Liu and Michaela U. Gack}

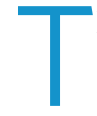
he continuous emergence of SARS-CoV-2 variants of concern (VOCs), from Alpha to Omicron, underscores the extraordinary capability of the virus to adapt to the human immune system. Extensive research has elucidated how changes in the viral spike protein, which mediates entry into cells, promote human-to-human spread and viral escape from antibody responses. By contrast, the role of mutations outside the spike protein in virus pathogenesis remains scarcely explored. In Nature, Krogan and colleagues ${ }^{1}$ crack the code of non-spike mutations found in the Alpha variant of SARS-CoV-2 by showing that some of these mutations ramp up the expression of viral innate immune antagonists, allowing escape from intrinsic immune defenses.

Alpha (Pango lineage: B.1.1.7 and Q lineages), which was first identified in the
UK and declared a VOC in December 2020, gained a substantial transmission advantage over earlier SARS-CoV-2 strains. While this superior performance is primarily due to specific spike mutations that enhance affinity to the viral entry receptor, angiotensin-converting enzyme 2 (ACE2), it has been unknown whether Alpha had learned new tricks once inside human cells. Intriguingly, Krogan and colleagues $^{1}$ find that Alpha replicates on the sly and stimulates antiviral responses much less efficiently than two first-wave isolates, indicating that Alpha is equipped with new mechanisms of immune evasion.

Type I and III interferons (IFNs) have key roles in virus restriction by upregulating a myriad of IFN-stimulated genes (ISGs) with antiviral or immunomodulatory properties. As a countermeasure, SARS-CoV-2 has evolved ways to suppress or dysregulate
IFN responses, a phenomenon observed both in vitro and also in patients critically ill with COVID-19 $9^{2,3}$. IFN induction is initiated by pattern-recognition receptors such as the retinoic acid-inducible gene I (RIG-I)-like receptors (RLRs), which detect RNA species of viral and host origins and are primary sensors of coronavirus infection ${ }^{4}$. Downstream of RLRs, TANK-binding kinase 1 (TBK1) and other kinases are activated, which then phosphorylate transcription factors (for example, IRF3 and IRF7) that drive the expression of IFNs and proinflammatory cytokines. Phosphoproteomics analysis revealed curtailed activities of these kinases early during infection with Alpha ${ }^{1}$, consistent with low IFN and ISG induction. Conversely, activation of these kinases at a later time was higher in cells infected with Alpha than in cells infected with the earlier 


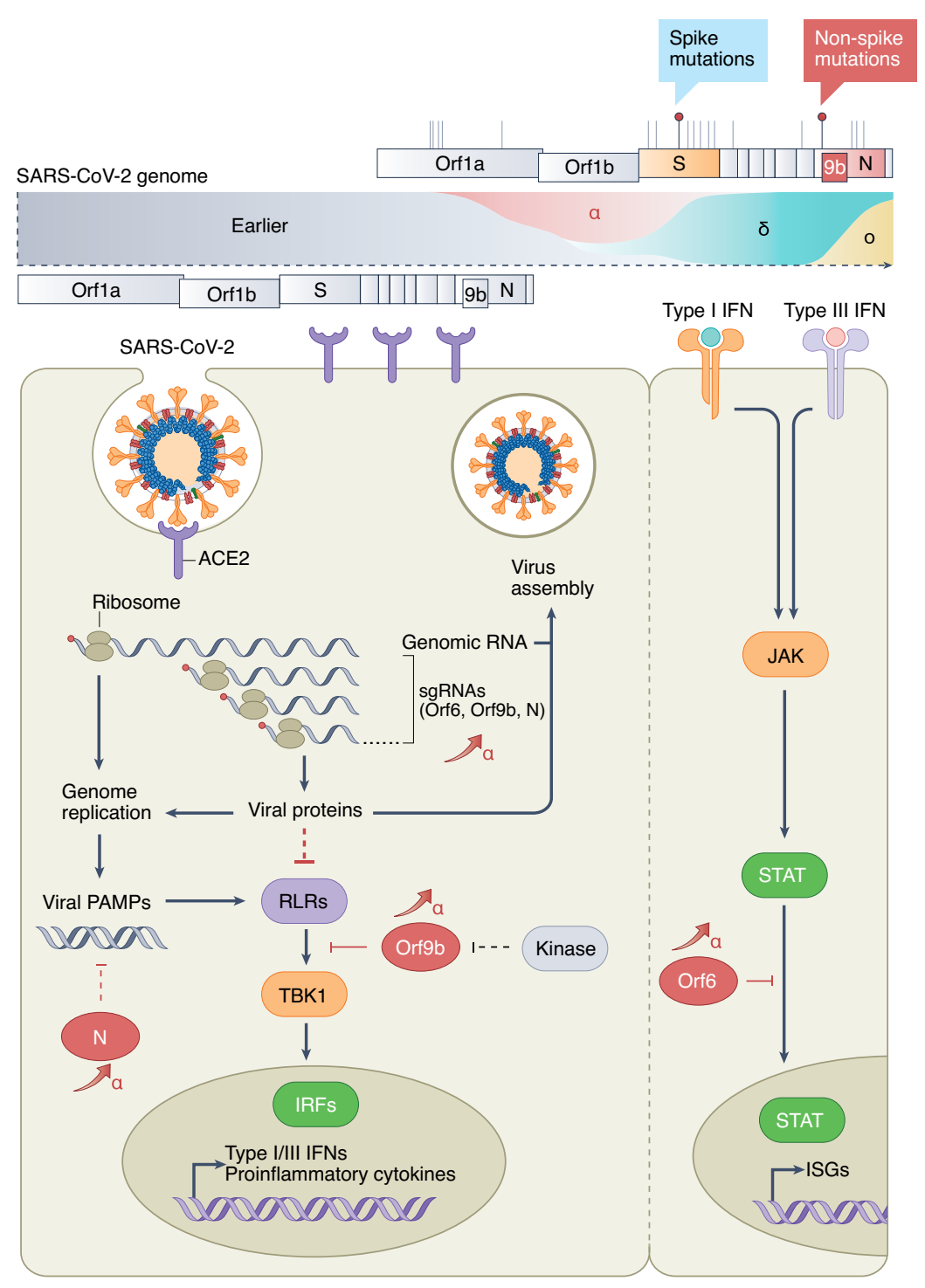

Fig. 1 | Non-spike mutations arm Alpha with new immune evasion tactics. Specific non-spike mutations allow Alpha to evade innate immune surveillance by upregulating the expression of viral sgRNAs and sgRNA-encoded IFN-inhibitory proteins (Orf6, Orf9b and N). Orf9b, whose IFN-antagonistic activity is counteracted by phosphorylation via an unknown cellular kinase, interacts with TOM70 and blocks the mitochondrial recruitment of TBK1 and IRF3, thereby suppressing IFN induction. N likely sequesters viral dsRNA species to shield them from RNA sensors such as RLRs. Orf6 inhibits the nuclear translocation of STATs and the ensuing ISG expression. Contextually similar nucleotide changes as those found in Alpha have been detected in recently emerged VOCs (Delta and Omicron), whose immunomodulatory effects are yet to be determined. ACE2, angiotensin-converting enzyme 2; sgRNA, subgenomic RNA; $\mathrm{N}$, nucleocapsid; RLRs, retinoic acid-inducible gene I (RIG-I)-like receptors; TBK1, TANK-binding kinase 1; IRFs, interferon-regulatory factors; JAK, Janus kinase; STAT, signal transducer and activator of transcription protein; ISG, interferon-stimulated gene; PAMPs, pathogen-associated molecular patterns.

isolates ${ }^{1}$, suggesting intricate mechanisms of kinase dysregulation by Alpha. However, defining the role of temporal kinase regulation in viral pathogenesis and the involvement of viral proteins and their regulation (as described below for Orf9b) requires further exploration. Furthermore, persistently lower proinflammatory responses were induced by Alpha, indicating that activation of nuclear factor- $\kappa \mathrm{B}(\mathrm{NF}-\kappa \mathrm{B})$ was also impaired. Whether this effect has any implication in the cytokine release syndrome observed in a proportion of patients remains to be fully determined.

Coronaviruses including SARS-CoV-2 bear the largest genomes among RNA viruses and encode nearly 30 proteins that together transform infected cells into a virus production factory. These viruses use a unique strategy for viral RNA synthesis that produces both full-length genomic RNA and a set of nested co-terminal subgenomic RNAs (sgRNAs) ${ }^{5}$. The sgRNAs encode, in addition to structural components, several accessory proteins that exhibit immunosuppressive activities (Fig. 1). By mining viral RNA sequencing and proteomics data, Krogan and colleagues find that Alpha produced greater amounts of sgRNA and sgRNA-encoded proteins such as Orf9b, Orf6 and nucleocapsid (N) than did earlier virus isolates ${ }^{1}$. This enhanced production is likely due to nucleotide changes that alter the transcriptional and/ or translational regulation of Orf9b and $\mathrm{N}^{1}$. Future work is needed to fully determine the mechanism(s) behind boosted sgRNA synthesis. It will also be important to further define the relevance of increased Orf9b and $\mathrm{N}$ sgRNAs to disease outcomes in human patients ${ }^{6}$.

Viral strategies of innate immune escape are diverse, and SARS-CoV-2 has evolved a wide variety of strategies to disarm innate host defenses. The viral methyltransferase and ribonuclease modify or cleave viral RNA to limit RLR sensing and ISG-dependent virus restriction ${ }^{7}$. The viral papain-like protease dampens innate immune signaling through direct de-ISGylation of melanoma differentiation-associated protein 5 (MDA5) and IRF $3^{8,9}$. The three SARS-CoV-2 proteins (Orf6, Orf9b and N) that were found to be heightened in expression during Alpha infection have also been shown to have IFN-antagonistic properties. Orf6 disrupts the nuclear translocation of the transcription factors STAT1 and STAT2, thereby inhibiting ISG expression ${ }^{10}$. Orf9b suppresses IFN induction by blocking mitochondrial recruitment of TBK1 and IRF3 through a mechanism that involves an interaction with translocase of outer membrane 70 $\left(\right.$ TOM70) ${ }^{11}$ (Fig. 1). Consistent with these viral mechanisms, Alpha infection led to stronger IRF and STAT inhibition than did wave-one isolates ${ }^{1}$. Interestingly, the ability of Orf9b to bind TOM70 and to suppress IFN responses was closely associated with Orf9b phosphorylation, suggesting that a hitherto unidentified cellular kinase regulates Orf9b-mediated inhibition of innate immunity. Furthermore, the authors find that Alpha infection rendered less immunostaining of intracellular double-stranded RNA (dsRNA) ${ }^{1}$, the well-known ligands for RLRs, suggesting another camouflage tactic used by Alpha. Whether this under-the-radar operation is because of lower dsRNA production 
or enhanced dsRNA masking (perhaps mediated by $\mathrm{N}$ ) remains to be fully determined (Fig. 1). Moreover, investigating the potential immunomodulatory effects of non-spike mutations in Alpha and the more recently emerged Omicron variant is an exciting avenue for future studies. Along these lines, whether any of these mutations help SARS-CoV-2 to evade detection by other sensors (for example, cGAS-STING and NOD-like receptors) remains to be determined.

The findings by Krogan and colleagues ${ }^{1}$ also demonstrate the need for a better understanding of SARS-CoV-2 genome evolution and human adaptation. Although spike mutations are still rapidly evolving, as shown by the emergence of the Omicron variant, it remains elusive how the rest of the viral genome co-evolves. Many of the non-spike mutations are unique, in contrast to spike mutations that are frequently shared among several VOCs (for example, D614G). The finding by the authors that recently emerged VOCs (that is, Delta and Omicron) contain contextually similar nucleotide changes as those altering transcriptional and/or translational regulation of Orf9b and $\mathrm{N}$ in the Alpha variant ${ }^{1}$ suggests that these mutations are more likely of functional than structural relevance. Future studies should focus on characterizing the roles of VOC-defining non-spike mutations in key virological and immunological aspects of SARS-CoV-2 disease. It will also be interesting to determine whether non-spike mutations act together with spike mutations to determine viral transmissibility and pathogenicity.

Genomic surveillance is important for identifying new SARS-CoV-2 variants and for predicting their evolutionary trajectories and pandemic potentials. The identification of Delta-Alpha recombinants, which are likely the consequence of a recombination event that conferred Delta the genomic portion of Alpha bearing sgRNA-enhancing mutations ${ }^{12}$, blows the whistle on the emergence of new variants with potentially more effective immune evasion strategies. A greater understanding of the link between the genetic composition of SARS-CoV-2 and its immunomodulatory properties may help design new antiviral therapies or immunomodulatory drugs that restore a functional antiviral IFN response.

GuanQun Liu (D) and Michaela U. Gack (D) $₫$ Cleveland Clinic Florida Research and Innovation Center, Port St. Lucie, FL, USA.

凶e-mail: gackm@ccf.org

Published online: 22 February 2022

https://doi.org/10.1038/s41590-022-01148-8

References

1. Thorne, L. G. et al. Nature https://doi.org/10.1038/s41586-021 04352-y (2021).

2. Blanco-Melo, D. et al. Cell 181, 1036-1045 (2020).

3. Hadjadj, J. et al. Science 369, 718-724 (2020).

4. Rehwinkel, J. \& Gack, M. U. Nat. Rev. Immunol. 20, 537-551 (2020).

5. V'Kovski, P., Kratzel, A., Steiner, S., Stalder, H. \& Thiel, V. Nat. Rev. Microbiol. 19, 155-170 (2021).

6. Parker, M. D. et al. Preprint at bioRxiv https://doi.org/10.1101/ 2021.03.02.433156 (2021)

7. Chan, Y. K. \& Gack, M. U. Nat. Rev. Microbiol. 14, 360-373 (2016).

8. Liu, G. et al. Nat. Microbiol. 6, 467-478 (2021).

9. Shin, D. et al. Nature 587, 657-662 (2020).

10. Miorin, L. et al. Proc. Natl Acad. Sci. USA 117, 28344-28354 (2020).

11. Gordon, D. E. et al. Science 370, eabe9403 (2020).

12. Sekizuka, T. et al. Preprint at medRxiv https://doi.org/10.1101/ 2021.10.11.21264606 (2021)

Competing interests

The authors declare no competing interests. 